\title{
Indications that maternal coxsackie B virus infection during pregnancy is a risk factor for childhood-onset IDDM
}

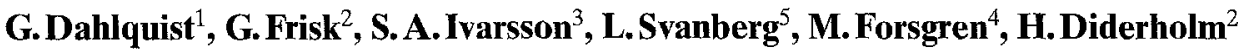 \\ ${ }^{1}$ Department of Pediatrics and Department of Epidemiology and Public Health, Umeå University, Umeå, Sweden \\ ${ }^{2}$ Research Unit of Replication Enzymology, University of Uppsala, Uppsala, Sweden \\ ${ }^{3}$ Department of Pediatrics, Malmö University Hospital, Malmö, Sweden \\ ${ }^{4}$ Department of Clinical Virology, Huddinge University Hospital, Karolinska Institute, Stockholm, Sweden \\ ${ }^{5}$ Department of Obstetrics and Gynecology, Malmö University Hospital, Malmö, Sweden
}

\begin{abstract}
Summary In a population-based setting, we traced serum samples collected at time of birth from $55 \mathrm{mo}-$ thers whose children later developed insulin-dependent diabetes (IDDM) and matched them pairwise to control subjects who gave birth at the same hospital during the same month. The sera were analysed for IgM antibodies to coxsackie $B$ virus serotypes 2,3 and $4(\mathrm{CBV}-2,3$ and 4$)$ using a type-specific $\mu$-antibody-capture radioimmunoassay. Despite a decreased power due to the close matching by time of birth we found a significantly higher frequency of CBV-3 IgM at delivery in mothers whose children later became diabetic compared to their matched control subjects. When using the presence of CBV-3 IgM as a risk factor the Mantel-Haenszel odds ratio estimate $(95 \%$ confidence limits) was $2.57(1.02 ; 7.31)$,
\end{abstract}

$p=0.043$. For CBV-2 and CBV-4, respectively no significant difference was found between mothers of patients and control subjects. According to the odds ratio estimate for CBV-3 and the proportion of exposed mothers among patients estimated in this study the aetiological fraction for this risk determinant would be $27 \%$. In conclusion, this study indicates that children of mothers who expressed CBV IgM at delivery are at increased risk for developing childhood onset IDDM. A fetal infection with CBV similar to rubella virus may initiate autoimmunity or cause persistent infection that may lead to progressive beta-cell destruction. [Diabetologia (1995) 38: 1371-1373]

Key words Pregnancy; Coxsackie B virus viral infections; Childhood IDDM.
Viral infections, especially due to picornaviruses, have long been suspected to be associated with insulin-dependent diabetes (IDDM) and evidence for such a mechanism has been collected in a large number of animal experiments. Viruses may directly infect and destroy the beta cells, as indicated from one case report where coxsackie B4 virus (CBV-4) was isolated from the pancreas of a child who died a few days after the onset of IDDM. Viruses may also more unspecifically precipitate disease onset by increasing the work load of an already damaged beta-

Received: 8 May 1995 and in revised form: 13 July 1995

Corresponding author: Dr. G.Dahlquist, Department of Pediatrics, Umeå University, S-901 85 Umeå, Sweden

Abbreviations: IDDM, Insulin-dependent diabetes mellitus; $\mathrm{CBV}$, coxsackie $\mathrm{B}$ virus; $\mathrm{CBV}-2,3,4$, coxsackie $\mathrm{B}$ virus serotypes 2,3 and 4 . cell, a mechanism which is suggested by the discovery of a dose-response relationship between the risk for IDDM and the frequency of recent infections. In humans, the most convincing evidence of virus as an early initiator of IDDM was the discovery of a very high prevalence of diabetes in a cohort of children who were followed-up because of fetal rubella embryopathy syndrome [1]. The rubella embryopathy syndrome is now virtually eradicated in Sweden due to vaccination programmes but other fetal virus infections might also affect the vulnerable immature immune system and thereby initiate autoimmunity to the beta cell.

In a recent population-based case-control study we found that mothers whose children later became diabetic had higher titres of group-specific enterovirus antibodies at time of giving birth when compared to mothers whose children were healthy, whereas no difference was found in antibody titres to herpes, 
Table 1. Pairwise distribution of mothers of diabetic and control children who showed IgM positivity/negativity against coxsackie $B$ virus serotypes 2,3 and 4 at delivery

\begin{tabular}{|c|c|c|c|c|c|c|c|c|c|}
\hline \multirow{3}{*}{$\begin{array}{l}\text { Diabetic } \\
\text { subjects }\end{array}$} & \multicolumn{3}{|l|}{$\mathrm{CBV}-2$} & \multicolumn{3}{|l|}{$\mathrm{CBV}-3$} & \multicolumn{3}{|l|}{$\mathrm{CBV}-4$} \\
\hline & \multicolumn{2}{|c|}{ Control subjects } & \multirow[t]{2}{*}{ Total cases } & \multicolumn{2}{|c|}{ Control subjects } & \multirow[t]{2}{*}{ Total cases } & \multicolumn{2}{|c|}{ Control subjects } & \multirow[t]{2}{*}{ Total cases } \\
\hline & positive & negative & & positive & negative & & positive & negative & \\
\hline Positive & 8 & 12 & 19 & 10 & 18 & 28 & 1 & 5 & 6 \\
\hline Negative & 9 & 26 & 36 & 7 & 20 & 27 & 10 & 35 & 45 \\
\hline Total control subjects & 17 & 38 & 55 & 17 & 38 & 55 & 11 & 40 & 51 \\
\hline
\end{tabular}

mumps and toxoplasmosis [2]. Enterovirus antibodies of low avidity to the antigens were observed indicating a recent infection but which of the more than 67 possible viruses that could react to the group-specific enterovirus assay was not elucidated in that study.

Since coxsackieviruses - belonging to the enterovirus group (a genus of the family picornaviridae) have specifically been one focus of interest in virus association to IDDM, we have analysed in the same population-based setting samples of sera from mothers whose children later became diabetic and in carefully matched control subjects type-specific IgM antibodies to coxsackie $B$ virus serotypes 2,3 and 4 (CBV-2, 3, 4).

\section{Subjects and methods}

The study was approved by the ethics committee of the Karolinska Institute. The approval was limited to analysis of anonymous sera; use of hospital records or questionnaires for clinical data of delivery was not permitted. Since 1 July 1977 all incident cases of IDDM in children aged 0-14 years are recorded in the Swedish Childhood Diabetes register with a level of ascertainment of $96-99 \%$ [3]. Through this register it was possible to trace all children who had developed diabetes up to the age of 15 years between 1 July 1977 and 1 January 1990 and who had been born at Malmö General Hospital after 1969. Since that year blood samples have been routinely taken from the mothers at time of giving birth, collected and stored frozen. Out of a total of 75 children belonging to this defined study who had become diabetic, 57 maternal sera were found [2]. To each of these sera, except two, a non-diabetic control subject was traced from the same serum bank and matched by month of birth; thus 55 matched pairs were analysed for IgM antibodies to $\mathrm{CBV}-2$ and 3 and 51 matched pairs for CBV-4 IgM.

A type-specific $\mu$-antibody-capture radioimmunoassay was used as previously described [4]. In this method polystyrene beads are coated with human-IgM - Fc - specific antibodies raised in rabbits (Jackson Immunoresearch Laboratory Inc., Baltimore, USA). Diluted serum were incubated with the coated beads for $2 \mathrm{~h}$ at $37^{\circ} \mathrm{C}$. The beads were then washed and ${ }^{35} \mathrm{~S}$-labelled virus was added. After overnight incubation the beads were washed and radioactivity counted. The results were expressed as reciprocal serum titres $(62.5,125$ etcetera up to 8,000 ). The cut-off point used was 3 times the value of the buffer blank and a negative serum (both gave the same $\mathrm{cpm}$ value), but with the proviso that the cutoff value should be at least $100 \mathrm{cpm}$. In this way borderline titres were also in- cluded [5]. The strains of CBV used as antigens and the procedures for their propagation, labelling and purification have also been previously described $[4,6]$.

\section{Statistical analysis}

Odds ratios as an estimate of the relative risk of a child developing IDDM when the mothers showed definite or borderline CBV-IgM titres were calculated using Mantel-Haenszel's method for matched analysis and the statistical significance was tested using McNemar's test. The $95 \%$ confidence limits were calculated using the test based method according to Miettinen. All the statistical methods have been described previously [7]. The calculations were performed using QUEST software (Gustafsson L., 1991. QUEST - A program system for statistical and epidemiological analysis. University of Umeå Press, Umeå, Sweden).

\section{Results}

In Table 1 the pairwise distribution of IgM positivity/ negativity for CBV-2, CBV-3 and CBV-4 is shown. A significantly higher frequency of CBV-IgM positivity was found for CBV-3 and a total of 28 out of the 55 mothers whose children later became diabetic had these antibodies. Out of the 55 matched pairs 25 were discordant and this yields a Mantel-Haenszel odds ratio estimate of 2.57 (95\% confidence limits $1.02 ; 7.31), p=0.043$. For CBV-2 the number of discordant pairs was 21 and the odds ratio 1.33 (confidence limits $0.52 ; 3.59$ ), $p=0.66$. For CBV-4 the number of discordant pairs was only 15 and the odds ratio 0.50 (confidence limits $0.13 ; 1.61$ ),$p=0.30$. Figure 1 shows the absolute frequencies of CBV-3 among diabetic and control subjects. If using the odds ratio estimate for CBV-3 and the proportion of exposed mothers among the cases found in this small study group to calculate the aetiological fraction

$=\frac{O R-1}{O R} \times$ proportion of exposed cases the aetiological fraction would be $27 \%$. 


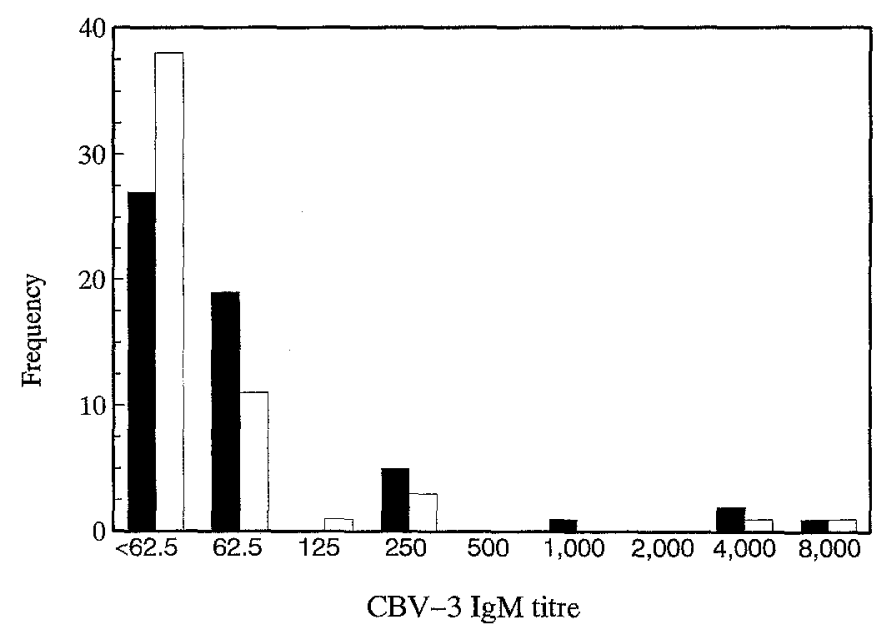

Fig. 1. Frequency distribution of $\mathrm{CBV}-3$ IgM antibodies among diabetic and control subjects $\square$

\section{Discussion}

The results of the present study indicate that within the group of enteroviruses shown to be associated with an increased risk for IDDM in children of mothers who were exposed during pregnancy [2], coxsackie B strains may be responsible. In the present study we chose to analyse CBV2, 3 and 4 only since previous Swedish studies on recent-onset IDDM cases have shown only these types to be of importance $[5,8]$. We used control subjects closely matched by time of birth to minimize the possibility of time-dependent confounders. This close matching by time will inevitably lead to an overmatching problem since we are studying an exposure which is epidemic in pattern i.e. associated with time. Thus, the matching has led to a loss of power in the present study since in the matched analysis only discordant pairs are effective and therefore the non-significant results for CVB-2 and CVB-4 may be due to this problem and thus a positive association with these types cannot be excluded. On the other hand, the statistically significant increase in odds ratio for CVB-3 may in fact be even stronger than the recorded 2.57.

The mechanism behind an association between fetal exposure to $\mathrm{CBV}$ and later development of IDDM may be either due to antigen mimicry or through association with a persistent viral infection, which is common with fetal and neonatal viral infections. Supporting the theory of direct antigen mimicry is the discovery of a striking sequence similarity between different coxsackie $B$ viruses and the beta-cell antigen glutamic acid decarboxylase [9] and also finding that children with newly diagnosed IDDM had antibodies to an epitope that was shown to crossreact with CBV and glutamic acid decarboxylase [10]. In the latter study only CBV-4 strains were analysed whereas Kaufman et al. [9] showed that other members of the CBV family also cross-reacted. The alter- native mechanism of action would be that of a persistent viral infection due to the fetal immune system having become tolerant to this early exposure. A persistent infection would permit the development of insulitis and a slowly progressive beta cell destruction by several possible mechanisms.

In conclusion, our study indicates, for the first time, that children of mothers who at delivery reveal coxsackie B IgM antibodies have an increased risk for developing IDDM before the age of 15 years. The magnitude of this potential risk factor when calculated as an aetiological fraction of disease occurrence in this material was as high as $27 \%$, indicating that a large number of cases may occur through an early exposure of CBV. If a vaccine specific for CBV could be developed, as recently indicated from animal experiments, an effective primary prevention strategy for childhood onset IDDM may become a reality.

Acknowledgements. This study was supported by grants from the Swedish Medical Research Council (project no.07531), the Nordic Insulin Foundation, The Swedish Diabetes Association and Lions.

\section{References}

1. Menser MA, Forrest JM, Bransby RD (1978) Rubella infection and diabetes mellitus. Lancet I: $57-60$

2. Dahlquist G, Ivarsson S, Lindberg B, Forsgren M (1995) Maternal enteroviral infection during pregnancy as a risk factor for childhood IDDM. Diabetes 44: 408-413

3. Dahlquist G, Mustonen L (1994) Childhood onset diabetes - time trends and climatological factors. Int J Epidemiol 23: $1234-1241$

4. Frisk G, Nilsson E, Ehrnst A, Diderholm H (1989) Enterovirus IgM detection using virions and procapsids of coxsackie B virus as antigen. J Virol Meth 24: 191-202

5. Frisk G, Friman G, Tuvemo T, Fohlman J, Diderholm H (1992) Coxsackie B virus IgM in children at onset of type 1 (insulin-dependent) diabetes mellitus: evidence for IgM induction by a recent or current infection. Diabetologia $35: 249-253$

6. Torfasson E, Frisk G, Diderholm H (1984) Indirect and reverse radioimmunoassays and their apparent specificites in the detection of antibodies of enteroviruses in human sera. J Med Virol 13: 13-31

7. Breslow NE, Day NE (1988) Statistical methods in cancer research, Vol 1. The analysis of case-control studies. IARC Scientific Publications, No.32, Lyon

8. Frisk C, Fohlman J, Kobbah M et al. (1985) High frequency of coxsackie-B-virus-specific IgM in children developing type 1 diabetes during a period of high diabetes morbidity. I Med Virol 17: 219-227

9. Kaufman DL, Erlander MG, Clare-Salzler M, Atkinson MA, MacLaren NK, Tobin AJ (1992) Autoimmunity to two forms of glutamate decarboxylase in insulin-dependent diabetes mellitus. J Clin Invest 89: 283-292

10. Laakkonen M, Hyöty H, Kulmala P et al. (1994) Evaluation of antibody binding to the common peptide epitope in $\mathrm{GAD}_{65}$ and coxsackie $\mathrm{B} 4$ virus $2 \mathrm{C}$ protein. 13th Diabetes Immunotherapy Workshop, Paris (Abstract) 